Article

\title{
Optimization Parameters of Trading System with Constant Modulus of Unit Return
}

\author{
Krzysztof Piasecki ${ }^{1, *(D)}$ and Michał Dominik Stasiak ${ }^{2}$ (D) \\ 1 Institute of Economy and Finance, WSB University in Poznań, ul. Powstańców Wielkopolskich 5, \\ 61-895 Poznań, Poland \\ 2 Department of Investment and Real Estate, Poznan University of Economics and Business, \\ al. Niepodleglosci 10, 61-875 Poznań, Poland; michal.stasiak@ue.poznan.pl \\ * Correspondence: krzysztof.piasecki@wsb.poznan.pl
}

Received: 29 June 2020; Accepted: 5 August 2020; Published: 18 August 2020

check for updates

\begin{abstract}
The unit return is determined as the return in the quotation currency (QCR) per the unit of base exchange medium (BEM). The main purpose is to examine the applicability of a trading system with a constant modulus of unit return (CMUR). The CMUR system supports speculative operations related to the exchange rate, given as the BEM quotation per the QCR. Premises for investment decisions are based on knowledge about the quotation dynamics described by its binary representation. This knowledge is described by a prediction table containing the conditional probability distributions of exchange rate increments. Any prediction table depends on observation range. Financial effectiveness of any CMUR system is assessed in the usual way by interest rate and risk index based on Shannon entropy. The main aim of our paper is to present algorithms which may be used for selecting effective CMUR systems. Required unit return modulus and observation range are control parameters applied for management of CMUR systems. Optimal values of these parameters are obtained by implementation of the proposed algorithm. All formal considerations are illustrated by an extensive case study linked to gold trading.
\end{abstract}

Keywords: speculative trading system; prediction table; financial effectiveness

\section{Introduction}

Each financial instrument is determined as a given asset in short position or in long position. The speculator's earning does not result from the underlying asset attributes, such as long-term technical analysis or fundamental ratios. In general, speculation is the acquisition of a financial instrument with the expectation that it will be sold as more expensive in the near future [1]. Therefore, speculation is acquisition of an asset in anticipation of its price increase, and also the disposal of an asset in anticipation of its price fall. For these reasons, speculation transaction is such financial transactions where the speculator's earnings result from short-term fluctuations in the quoted price of a traded asset. For these reasons, speculation transactions can only be implemented on financial markets characterized by frequent price changes. Moreover, the duration of a single speculative transaction is generally short. This is why speculation transactions require the use of High-Frequency Trading (HFT) systems. HFT systems help traders hold positions for short periods of time and earn their profits by accumulating tiny gains on a large number of transactions [2].

The existing body of literature on HFT is extensive, and it covers many items devoted to different aspects of HFT [2,3]. IT techniques' development implies an increase in HFT systems' popularity.

The subject of speculation is trading in base exchange medium (BEM), given as any currency, any precious metal, or any standardized commodity. All speculative transactions are related to an exchange rate, given as the BEM quotation per the quotation currency (QCR). The trading in 
exchange rates is organized on the Foreign eXchange market (FX), characterized by very frequent price changes. Therefore, speculative transactions are implemented on FX. A price dynamic on FX may be characterized by unite return, defined as the return in QCR per unit of BEM [4].

Generally, financial speculators use well-known methods to manage speculative transactions. On the other hand, most speculators bear losses $[5,6]$. The above fact shows that this is not a good approach to profitable trading. Hence, there is demand for a new, fully justified method of financial market analyses supporting speculative transactions [7].

Among other things, heuristic trading systems are used on financial markets [8,9]. On the other hand, applied mathematics is the obvious research field for new HFT systems. For example, here, we use advanced mathematical statistics [10-12], genetic algorithms [13-15], artificial intelligence models [11], neural networks [16-21], multi agent theory [10,22,23], and mathematics for fuzzy systems [10]. Applying these theories requires acceptance of strong formal assumptions. Therefore, the mathematical apparatus used significantly limits the field of practical applications. This causes a demand for such HFT systems that their mathematical models are based on weak formal assumptions.

An HFT system may be determined with use of the criterion of Constant Modulus of Return Rate (CMUR) [4]. To the best of our knowledge, proposing CMUR systems in 2019 is completely original. For this reason, the CMUR systems' bibliography contains only Reference [4] and a few pilot studies [24-32].

The correct application of the CMUR system requires only a few general assumptions. Moreover, the CMUR system is very basic. Therefore, applied mathematical apparatus does not significantly restrict the area of CMUR system use. In previous pilot studies [23-32], the necessary mathematical assumptions were always met, and this is a very important advantage of the CMUR system.

Aldridge [7] distinguish the following FX participants: high-frequency speculators, long-term investors, and corporations. The CMUR system can support speculators. Any HFT system can consist of three components: transaction management, risk reduction, and money management [33,34]. The CMUR system does not refer to money management, which is a disadvantage of the CMUR system.

Any HFT system is related to the method of financial prediction [2,10,35-39]. In the CMUR system, a sign of unitary return is forecasted with the use of a simple prediction table specified for required unitary return modulus on the space of all binary states of an assumed observation range. In this way, required unitary return modulus and assumed states observation range are the only control parameters of the CMUR system. It means that any CMUR system is uniquely determined by the fixed pair of control parameters.

The application of the CMUR system each time requires choice of the optimal variant of its control parameters. This selection is made by the speculator. The main goal of this paper is presentation of a method of optimization control parameters of the CMUR system. Any variant of control parameters will be evaluated by the ex-ante characteristic proposed in Reference [4]. Optimal variants of the control parameters system will be chosen as the Pareto set.

The article is organized in the following way. Section 2 describes the procedure of discretization Ask price trend. Here, we simplify the procedure description given in Reference [4]. We obtained this simplification in this way by omitting all the proofs and justifications required in Reference [4]. In an analogous way, we simplified the description of the CMUR model, as presented in Section 3. In Section 4, we present some tools for assessing financial effectiveness of CMUR systems. In Section 5, we propose original algorithms that select the optimal variants of the CMUR system. In Section 6, obtained theoretical results are illustrated by an extensive case study linked to gold trading. Section 7 justifies the ambiguity of the obtained results and points to the future research directions.

\section{Discretization of Ask Price Trend}

By basic exchange medium (BEM), we understand any base currency, any precious metal, or any standardized commodity. On the financial market of the exchange pair BEM/QCR, each considered BEM price is expressed in the quoted currency (QCR). The exchange pair BEM/QCR market is also 
called the BEM/QCR market. The Ask price of BEM is the unit price of BEM in the QCR. In each moment of time, $t \in[0, T]$, we note the Ask price, $Q_{A s k}(t)$. In this way, we determine the Ask price trend, $Q_{A s k}:[0, T] \rightarrow \mathbb{R}^{+}$, which describes the dynamics of an exchange pair, BEM/QCR.

In this section, we describe such method of discretization Ask price trend, which is dedicated to CMUR systems. This discretization method was introduced and justified in Reference [4]. The changes on the BEM/QCR market are described by a unit return, defined as a dirty return by the BEM amount. The unit return $u r\left(t^{\prime}, t^{\prime \prime}\right)$ in the time interval $\left[t^{\prime}, t^{\prime \prime}\right]$ equals

$$
u r\left(t^{\prime}, t^{\prime \prime}\right)=Q_{A s k}\left(t^{\prime \prime}\right)-Q_{A s k}\left(t^{\prime}\right) .
$$

In the first step, we create a binary representation of Ask price trend. Applied binary representation is inspired by the historical point-symbolic notation [40].

We observe a continuous trend, $Q_{A s k}:[0, T] \rightarrow \mathbb{R}^{+}$. If a single trend observation begins at the time $t^{\prime}$, then it ends as early as possible at the moment $t^{\prime \prime}>t^{\prime}$, fulfilling condition

$$
\left|u r\left(t^{\prime}, t^{\prime \prime}\right)\right|=\delta>0
$$

where $\delta$ is assumed as the discretization unit. We must remember that the assumed discretization unit, $\delta$, should be equal to the unit return modulus requested by the speculator.

The event, $\mathscr{I}_{\delta}$, denotes such an Ask price increase which fulfils the condition (2). The complement, $\mathscr{I}_{\delta}^{\mathrm{C}}$, of this event is Ask price fall fulfilling condition (2). Forecasting changes in the Ask price is limited to answering the question of whether there will be an increase, $\mathscr{I}_{\delta}$, or a decrease, $\mathscr{F}_{\delta}^{C}$, after the current time, $t^{\prime}$. The examined CMUR systems require only such elementary forecasts.

The first trend observation begins at the time $t_{0}=0$. We assume that the observation continuity of it means that at each moment of closing the observation, the next observation is opened. That is why the sequence, $\left(t_{i}\right)_{i=0}^{n} \subset[0, T]$, of observation opening moments is recursively determined in the following way:

$$
\begin{aligned}
t_{0} & =0, \\
t_{i+1}=\min \left\{\operatorname { m i n } \left\{\tau>t_{i}: u \mu\left(t_{i}, \tau\right)\right.\right. & \left.=\delta\}, \min \left\{\tau>t_{i}: u \mu\left(t_{i}, \tau\right)=-\delta\right\}\right\} .
\end{aligned}
$$

The sequence $\left(t_{i}\right)_{i=0}^{n}$ is a record of the observation history. Therefore, the sequence $\left(t_{i}\right)_{i=0}^{n}=\Theta$ is called an observation record.

The binary representation of the trend $Q_{A s k}:[0, T] \rightarrow \mathbb{R}^{+}$consists of transforming this trend into its binary representation $\mathcal{E}=\left(\varepsilon_{i}\right)_{i=1}^{n}$, determined as follows:

$$
\varepsilon_{i}=\left\{\begin{array}{cc}
1 & u r\left(t_{i-1}, t_{i}\right)=\delta \\
0 & u r\left(t_{i-1}, t_{i}\right)=-\delta
\end{array} .\right.
$$

The number, $n$, of binary observations contained in the binary representation $\mathcal{E}=\left(\varepsilon_{i}\right)_{i=1}^{n}$ depends on the discretization unit $\delta$ and the length $T$ of the observation interval.

For set observation range $c \in \mathbb{N}$, we define the state space $\mathbb{E}_{c}$ as the set of all $c$-elements' permutations with repetition from the set $\{0,1\}$. The state space $\mathbb{E}_{c}$ consists of $2^{c}$ states defined in the following way:

$$
\mathrm{s}_{j}=\left(e_{1}, e_{2}, \ldots, e_{c}\right),
$$

where

$$
j=\sum_{l=1}^{c} 2^{c-l} e_{l}+1
$$

The observation range $c \in \mathbb{N}$ is chosen by an analyst examining the BEM/QCR market. We will use any state $s_{j} \in \mathbb{E}_{c}$ as as the only premise for the forecast required by the CMUR system. 
Example 1 [4]. For the number $c=4$, we have the following state space:

$$
\mathbb{E}_{4}=\left\{\begin{array}{c}
s_{1}=(0,0,0,0), s_{2}=(0,0,0,1), s_{3}=(0,0,1,0), s_{4}=(0,0,1,1), \\
s_{5}=(0,1,0,0), s_{6}=(0,1,0,1), s_{7}=(0,1,1,0), s_{8}=(0,1,1,1), \\
s_{9}=(1,0,0,0), s_{10}=(1,0,0,1), s_{11}=(1,0,1,0), s_{12}=(1,0,1,1), \\
s_{13}=(1,1,0,0), s_{14}=(1,1,0,1), s_{15}=(1,1,1,0), s_{16}=(1,1,1,1),
\end{array}\right\} .
$$

Let us take into account fixed binary representation $\mathcal{E}=\left(\varepsilon_{i}\right)_{i=1}^{n}$ associated with observation record $\Theta$. Each moment $t_{i} \in \Theta(i=c, c+1, \ldots, n)$ is attributed to an observed state, $\tilde{s}_{i} \in \mathbb{E}_{c}$, of the $\mathrm{BEM} / \mathrm{QCR}$ market. In this way, we create a sequence,

$$
\Xi=\left(\tilde{s}_{i}\right)_{i=c}^{n}=\left(\left(\varepsilon_{k}\right)_{k=i-c+1}^{i}\right)_{i=c^{\prime}}^{n}
$$

of following observations of market states. The sequence, $\Xi$, defined in this way is a record of all observed changes in BEM/QCR market states. Therefore, the sequence, $\Xi$, is called a state record.

For any pair, $\left(t_{i}, t_{i+1}\right) \subset \Theta$, the value $\varepsilon_{i+1}=1$ denotes the observation of rise in the Ask price in the interval $\left[t_{i}, t_{i+1}\right]$. Then, the value $\varepsilon_{i+1}=0$ denotes the observation of fall in the Ask price in the interval $\left[t_{i}, t_{i+1}\right]$. For the purposes of Ask price trend discretization, the sequence

$$
\Psi=\left(\tilde{s}\left(t_{i}\right), \varepsilon_{i+1}\right)_{i=c}^{n-1}
$$

plays the role of a training dataset [41].

Each state, $s_{j} \in \mathbb{E}_{c}$, is observed exactly $n_{j}$ times. The sequence $\left(n_{j}\right)_{j=1}^{2^{c}}$ of random numbers is explicitly determined by training dataset $\Psi$. Thanks to this, for each $j=1,2, \ldots, m$, we can establish the probability

$$
p\left(s_{j}\right)=\frac{n_{j}}{n-c-1}
$$

of achieving the state $s_{j}$. Moreover, the training dataset $\Psi$ determines the space of all observed states

$$
\mathbb{G}_{c}(\Psi)=\left\{s_{j} \in \mathbb{E}_{c}: n_{j}>0\right\} .
$$

In Reference [4] it is justified that the training dataset $\Psi$ uniquely determines conditional probability

$$
p\left(\mathscr{I}_{\delta} \mid s_{j}\right)=\frac{n_{j}^{*}}{n_{j}},
$$

of achieving assumed rise, $\mathscr{I}_{\delta}$, immediately after that the state, $s_{j}$, has occurred. The sequence $\left(n_{j}^{*}\right)_{j=1}^{m}$ is explicitly determined as follows:

$$
n_{j}^{*}=\sum_{\left\{i: c \leq i \leq n-c-1, \tilde{s}_{i}=s_{j}\right\}} \varepsilon_{i}
$$

The total probability, $p\left(\mathscr{J}_{\delta}\right)$, of rise in Ask price can be determined as:

$$
p\left(\mathscr{I}_{\delta}\right)=\sum_{s_{j} \in \mathbb{G}_{c}(\Psi)} p\left(s_{j}\right) \cdot p\left(\mathscr{J}_{\delta} \mid s_{j}\right)
$$

The set of triples below:

$$
\mathcal{P}(\mathrm{BEM} / \mathrm{QCR}, \delta, c, \Psi)=\left\{\left(n_{j}, p\left(s_{j}\right), p\left(\mathscr{J}_{\delta} \mid s_{j}\right)\right): s_{j} \in \mathbb{G}_{c}(\Psi)\right\},
$$


forms the prediction table to foresee the changes of the BEM/QCR exchange rate For any exchange pair $\mathrm{BEM} / \mathrm{QCR}$, its prediction table depends only on three parameters:

- $\Psi$-training dataset,

- $\delta$-discretization unit,

- $\quad c$-observation range.

The training dataset $\Psi$ depends on observed ex-ante Ask price trend, $Q_{A s k}:[0, T] \rightarrow \mathbb{R}^{+}$, on required discretization unit, $\delta$, and on assumed observation range, $c$. During the calculation of the prediction table, financial analysts and traders do not have influence on the observed ex-ante Ask price trend. Therefore, training dataset $\Psi$ cannot be taken into account as a control parameter of a prediction table.

On the other hand, discretization unit, $\delta$, and observation range, $c$, are always specified by financial analysts and traders. Therefore, these last two parameters may be considered as control ones.

The confidence interval of a sample statistic (13) is given as the Wald confidence interval [42]. The proposed approximation is reliable when the sample size is large enough, and the success probability is noticeably different from 1 [43].

The value $\alpha$ is the assumed value of a significance level. For each state, $s_{j} \in \mathbb{G}_{c}(\Psi)$, the $(1-2 \cdot \alpha)$ level Wald's confidence interval is given by the equation

$$
\mathbb{W}_{j}(\alpha)=\left[p\left(\mathscr{J}_{\delta} \mid s_{j}\right)-z_{\alpha} \cdot \sqrt{\frac{p\left(\mathscr{I}_{\delta} \mid s_{j}\right)\left(1-p\left(\mathscr{J}_{\delta} \mid s_{j}\right)\right)}{n_{j}}}, p\left(\mathscr{J}_{\delta} \mid s_{j}\right)+z_{\alpha} \cdot \sqrt{\frac{p\left(\mathscr{J}_{\delta} \mid s_{j}\right)\left(1-p\left(\mathscr{I}_{\delta} \mid s_{j}\right)\right)}{n_{j}}},\right]
$$

where $z_{\alpha}$ is the $\alpha\{\backslash$ displaystyle $1-\{\backslash$ tfrac $\{\backslash$ alpha $\}\{2\}\}\}$ quantile of a standard normal distribution. In financial practice, the minimal sample size should be determined empirically.

Each state, $s_{j} \in \mathbb{E}_{c}$, can use a prediction premise. If prediction premise $s_{j} \in \mathbb{E}_{c}$ fulfils the condition

$$
\left\{1-p\left(\mathscr{J}_{\delta} \mid s_{j}\right)\right\} \notin \mathbb{W}_{j}(\alpha)
$$

then it is called a well-justified premise. Other prediction premises are called ill-justified ones. In this way, for assumed $\alpha$ level of significance, we determine the space,

$$
\mathbb{D}(\alpha, \delta, c)=\left\{s_{j} \in \mathbb{G}_{c}(\Psi):\left\{1-p\left(\mathscr{J}_{\delta} \mid s_{j}\right)\right\} \notin \mathbb{W}_{j}(\alpha)\right\},
$$

of all well-justified premises. For any well-justified premise, the probability of inaccurate forecasting Ask price change is not greater than the value $\alpha$. For ill-justified premises, this probability increases. Therefore, when it is possible, ill-justified premises should be avoided.

In practice, FX is the main financial market where the BEM/QCR exchange rate is traded. All quotations on the FX are related to the BEM unit. For any base currency (BCR) except Japanese Yen (JPY), the BEM unit equals to its monetary unit. For JPY, the BEM unit is equal to 100 JPY. For any precious metal, the BEM unit is equal to 1 troy ounce $(1 \mathrm{oz})$. Major precious metals traded in the FX are gold, marked by acronym XAU, and silver, marked by acronym XAG.

On the FX, traders use the lot as a measure unit for the BEM amount. Table 1 contains different lot values determined for various BEMs.

Table 1. Lot definitions for main base exchange mediums (BEMs).

\begin{tabular}{cc}
\hline BEM & Lot Size \\
\hline BCR & $100,000 \mathrm{BEM}$ units \\
XAU & $100 \mathrm{oz}$ \\
XAG & $1000 \mathrm{oz}$ \\
\hline
\end{tabular}


In FX, a change in an exchange rate is expressed in pips. One pip is equal to 0.0001 lot of QCR.

Example 2 [4]. We discuss some cases of speculations in exchange pair silver quoted in U.S. dollars (XAG/USD). The selected value, $\delta=28$ pips, of assumed discretization unit is justified in Reference [28]. We use the space $\mathbb{E}_{4}$ of all possible FX states.

In the first research stage, we determine the prediction table and distinguish well-justified premises. The Dukascopy broker has collected tick data containing 42,782,438 XAG/USD quotations from 31 December 2012 to 1 January 2018, denoted in the following way: [0, 5Y]. For exchange pair XAG/USD, these tick data determine the Ask price trend, $Q_{A s k}:[0,5 Y] \rightarrow \mathbb{R}^{+}$. This trend graph is presented in Figure 1.

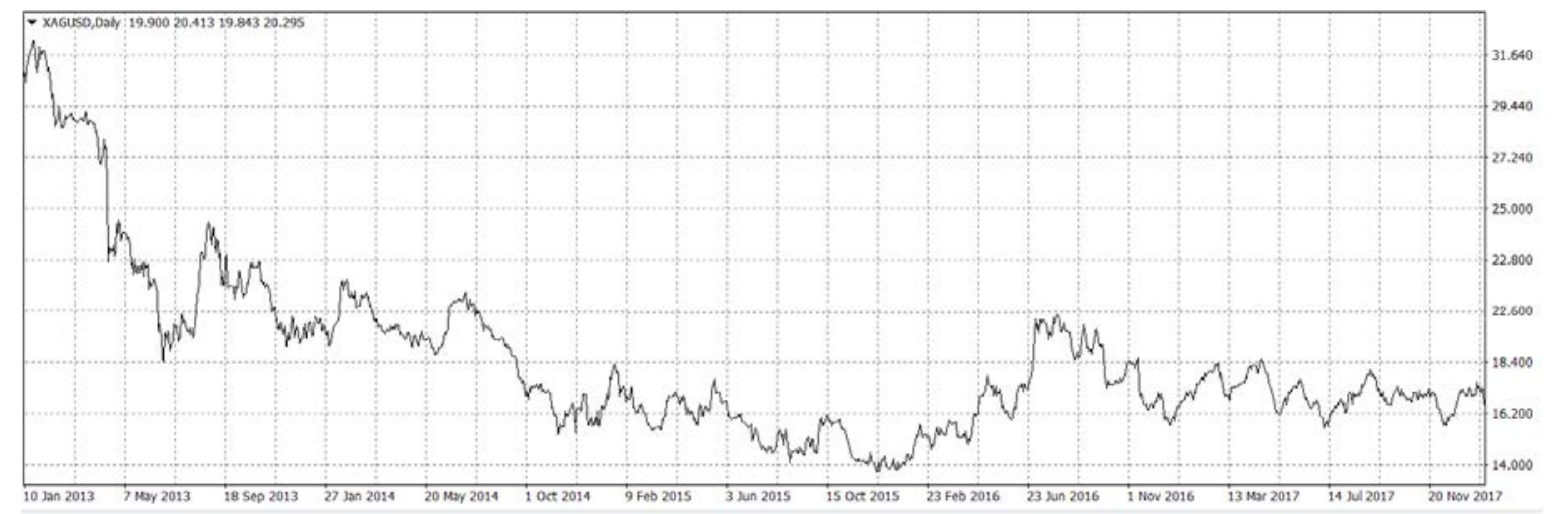

Figure 1. The graph for trend of Ask price of XAG/USD exchange pair.

We see that the trend $Q_{\text {Ask }}$ contains descriptions of bull market, bear market rand, and sideways market. For this reason, we can say that collected tick data are representative.

Using recurrent Formula (4), we transform the collected tick data into the observation record $\Theta=\left(t_{i}\right)_{i=0}^{1480} \subset$ $[0,5 Y]$. In the next step, with Formula (5), we set binary representation $\mathcal{E}=\left\{\varepsilon_{i}\right\}_{i=1}^{1480}$ of Ask price trend $Q_{\text {Ask }}$.

The binary representation $\mathcal{E}$ is applied for determination of the state record $\Xi=\left(\tilde{s}_{i}\right)_{i=4}^{1480}=\left(\left(\varepsilon_{k}\right)_{k=i-3}^{i}\right)_{i=4}^{1480}$. Taking together all the above results, we build training dataset $\Psi=\left(\widetilde{s}\left(t_{i}\right), \varepsilon_{i+1}\right)_{i=4}^{1479}$, which is used for calculation of the prediction table $\mathcal{P}(X A G / U S D, 28,4, \Psi)$ presented in Table 2. For significance level $\alpha=0.05$, we determine Wald's confidence intervals $\mathbb{W}_{j}(0.05)$ by using Formula (17). Then, we distinguish the space $\mathbb{D}(0.05,28,4)$ of all well-justified premises. In Table 2, the ill-justified premises are market by the hash, \#. The last row in Table 2 shows the following: a total number of observations $n-5$ and a total probability $p\left(\mathscr{I}_{28}\right)$ of assumed rise in Ask price. The probability, $p\left(\mathscr{I}_{28}\right)$, is calculated using Formula (15).

For premises $s_{2}, s_{3}, s_{4}, s_{5}, s_{6}, s_{8}, s_{10}, s_{11}, s_{13}$, and $s_{14}$, the probability of inaccurately forecasting Ask price change is less than or equal to $\alpha=0.05$. In the next stage of the research, we will examine the profitability of financial decisions made under the impact of well-justified premises. This topic will be discussed in Example 3. 
Table 2. Prediction table $\mathcal{P}(X A G / U S D, 28,4, \Psi)$ and Wald confidence intervals.

\begin{tabular}{ccccc}
\hline$s_{j}$ & & $p\left(s_{j}\right)$ & $p\left(\mathscr{J}_{28} \mid s_{j}\right)$ & $\mathbb{W}_{j}(\mathbf{0 . 0 5})$ \\
\hline$s_{1}^{\#}$ & 96 & 0.065085 & 0.4688 & {$[0.385272,0.552328]$} \\
$s_{2}$ & 87 & 0.058983 & 0.4205 & {$[0.333705,0.507295]$} \\
$s_{3}$ & 101 & 0.068475 & 0.5941 & {$[0.513965,0.674235]$} \\
$s_{4}$ & 77 & 0.052203 & 0.4487 & {$[0.355745,0.541655]$} \\
$s_{5}$ & 88 & 0.059661 & 0.5730 & {$[0.486524,0.659476]$} \\
$s_{6}$ & 117 & 0.079322 & 0.5897 & {$[0.515121,0.664279]$} \\
$s_{7}^{\#}$ & 92 & 0.062373 & 0.5000 & {$[0.414509,0.585491]$} \\
$s_{8}$ & 82 & 0.055593 & 0.4146 & {$[0.325377,0.503823]$} \\
$s_{9}^{\#}$ & 88 & 0.059661 & 0.4886 & {$[0.401210,0.575998]$} \\
$s_{10}$ & 91 & 0.061695 & 0.4505 & {$[0.364963,0.536037]$} \\
$s_{11}$ & 117 & 0.079322 & 0.5897 & {$[0.515121,0.664279]$} \\
$s_{12}^{\#}$ & 95 & 0.064407 & 0.4947 & {$[0.410570,0.578825]$} \\
$s_{13}$ & 89 & 0.060339 & 0.4444 & {$[0.358019,0.530781]$} \\
$s_{14}$ & 84 & 0.056949 & 0.4167 & {$[0.328481,0.504919]$} \\
$s_{15}^{\#}$ & 81 & 0.054915 & 0.4634 & {$[0.372533,0.554267]$} \\
$s_{16}^{\#}$ & 90 & 0.061017 & 0.5111 & {$[0.424686,0.597514]$} \\
Total & 1475 & - & 0.4973 & \\
\hline
\end{tabular}

Source: Own elaboration.

\section{Description of the CMUR System}

The CMUR system is designed to support trading on FX. Trading on FX is managed by a broker. The applied variant of the CMUR system is selected by a speculator. Any exchange pair BEM/QCR is traded on FX in the following way.

The current value of BEM is quoted in two prices: The Bid price and the Ask price. Bid reflects how much of QCR will be obtained when we dispose of the BEM unit. Observed evolution of these prices is described by trends $Q_{A s k}:[0, T] \rightarrow \mathbb{R}^{+}$and $Q_{B i d}:[0, T] \rightarrow \mathbb{R}^{+}$. Ask price is always greater than Bid price. Therefore, we have

$$
Q_{A s k}(t)-Q_{B i d}(t)=\operatorname{spr}(t)>0 .
$$

The difference, spr: $[0, T] \rightarrow \mathbb{R}^{+}$, between a current Ask and Bid price is determined by the broker. This difference is called spread.

Two types of transaction may be opened on FX: long position and short position. A long position is opened by BUY order and it is closed by SELL order. A short position is opened by SELL order and it is closed by BUY order. The BUY order is made with the Ask price, $Q_{A s k}$, and it means acquisition BEM with QCR. The SELL order is made with the Bid price, $Q_{B i d}$, and it means disposal BEM with QCR. Transactions on FX are concluded between brokers and speculators. At FX, only the speculator can give the broker an order to BUY or SELL. A broker accepting an opening order is obliged to accept the closing order. The amount of the order is defined by the speculator.

Each transaction consists of its opening in time $t^{\prime}$ and its closing in a time $t^{\prime \prime}>t^{\prime}$. On the FX, the duration of the transaction is short. That is why we assume

$$
\forall_{\tau \in\left[t^{\prime}, t^{\prime \prime}\right]}: \operatorname{spr}(\tau)=\operatorname{spr}\left(t^{\prime}\right)=\overline{s p r}>0 .
$$

This condition is almost always satisfied. The value of a spread, $\overline{s p r}$, depends on the offer of the broker. Therefore, we cannot take spread, $\overline{s p r}$, into account as a control parameter of the CMUR system. On the other hand, the individual variants of the CMUR system differ in the value $\delta>\overline{s p r}$ of the required unit return modulus, which is always specified by the speculator. Therefore, unit return 
modulus may be considered as a control parameter of the CMUR system. In Reference [4], these last two parameters are used for determining the following ratio:

$$
\pi_{u p}=\frac{\delta+\overline{s p r}}{2 \cdot \delta}>\frac{1}{2}
$$

which will be interpreted later below the formula (27).

FX brokers set their fees based on commission equal to the spread. A speculator wants to earn due to an accurate prediction of a BEM/QCR quotation change. To achieve this goal, a speculator can apply the CMUR system. A CMUR system is linked to the prediction table $\mathcal{P}(\mathrm{BEM} / \mathrm{QCR}, \delta, c, \Psi)$, where the discretization unit, $\delta$, is equal to the unit return modulus required by the speculator. Since the discretization unit, $\delta$, and the observation range, $c$, are the only control parameters of the prediction table $\mathcal{P}(\mathrm{BEM} / \mathrm{QCR}, \delta, c, \Psi)$, the unit return modulus, $\delta$, and the observation range length, $c$, are control parameters of any CMUR system. In Reference [4], it is shown that these last two parameters are the only control ones of the CMUR system.

The effectiveness of CMUR system operation results from the proper selection of transaction premises. Each well-justified premise can be used as a premise for opening transaction on FX. Using the ratio (22), we distinguish the following kind of transaction premises. If well-justified transaction premise $s_{j} \in \mathbb{D}(\alpha, \delta, c)$ fulfils the condition

$$
\pi\left(s_{j}\right)=\max \left\{p\left(\mathscr{I}_{\delta} \mid s_{j}\right), 1-p\left(\mathscr{I}_{\delta} \mid s_{j}\right)\right\}>\pi_{u p},
$$

then it is called an acceptable transaction premise [4]. The value $\pi\left(s_{j}\right)$ will be explained belowthe Formula (27). In this way, we determine the space

$$
\mathbb{D}=\mathbb{D}\left(\alpha, \delta, c, \pi_{u p}\right)=\left\{s_{j} \in \mathbb{D}(\alpha, \delta, c): \pi\left(s_{j}\right)>\pi_{u p}\right\}
$$

of all well-justified premises. If we open a transaction after encountering an acceptable premise, then the probability of a loss is relatively low. For non-acceptable premises, this probability increases. We can expect that transactions opened repeatedly under acceptable premises will be profitable. Therefore, non-acceptable transaction premises should be avoided.

Example 3 [4]. Let us consider some ways of speculating on the XAG/USD exchange market. The speculator plans to speculate only using transactions with the unit return modulus $\delta=28$. These speculations may be supported by using prediction table $\mathcal{P}(X A G / U S D, 28,4, \Psi)$, presented in Table 2. Using (23), for each well-justified premise, we calculate its success probability, presented in Table 3. For the pair XAG/USD, the broker offers the spread $\overline{\text { spr }}=1$ pip. Therefore, we have the infimum of acceptable success probability, $\pi_{u p}=0.5179$. It, together with condition (23), implies that all well-justified transaction premises are acceptable.

Table 3. Strategy given by constant modulus of unit return $(C M U R)(\mathcal{P}(X A G / U S D, 28,4, \Psi), 1,0.05)$.

\begin{tabular}{cccc}
\hline Acceptable Premise $s_{j}$ & Probability of Success $\pi\left(s_{j}\right)$ & Recommendation & Expected Single Payment $\boldsymbol{Y}\left(s_{j}\right)$ \\
\hline$s_{2}$ & 0.5795 & SELL & $\$ 3.452$ \\
$s_{3}$ & 0.5941 & SELL & $\$ 4.270$ \\
$s_{4}$ & 0.5513 & SELL & $\$ 1.873$ \\
$s_{5}^{*}$ & 0.5730 & BUY & $\$ 3.088$ \\
$s_{6}^{*}$ & 0.5897 & BUY & $\$ 4.023$ \\
$s_{8}^{*}$ & SELL & $\$ 3.782$ \\
$s_{10}$ & 0.5854 & SELL & $\$ 1.772$ \\
$s_{11}^{*}$ & 0.5495 & BUY & $\$ 4.023$ \\
$s_{13}$ & 0.5897 & SELL & $\$ 2.114$ \\
$s_{14}$ & 0.5556 & SELL & $\$ 3.665$ \\
\hline
\end{tabular}

Source: own calculations. 
We can expect that transactions opened repeatedly under premises $s_{2}, s_{3}, s_{4}, s_{5}, s_{6}, s_{8}, s_{10}, s_{11}, s_{13}$, and $s_{14}$ will be profitable. For these premises, the probability of opening a profitable transaction is greater than or equal to $1-\alpha=0.95$.

Each trading system is linked to the implemented strategy of transaction-making. The CMUR system is related to a very simple strategy with only the following four transaction-making rules:

- Any transaction can be opened only at the moment $t^{\prime}$, when the acceptable premise, $s_{j} \in \mathbb{D}$, has occurred.

- If, for observed premise $s_{j}$, we have

$$
p\left(\mathscr{I}_{\delta} \mid s_{j}\right)>\frac{1}{2}
$$

then place a BUY order and go long.

- If, for observed premise $s_{j}$, we have

$$
p\left(\mathscr{I}_{\delta} \mid s_{j}\right)<\frac{1}{2}
$$

then place a SELL order and go short.

- Each opened transaction is closed at the moment $t^{\prime \prime}$, determined by dependence

$$
t^{\prime \prime}=\min \left\{\tau: \tau>t^{\prime},\left|u\left(t^{\prime}, \tau\right)\right|=\delta\right\}
$$

Listing the applied transaction-making rules ends the CMUR system description. We denote the CMUR system described in this section by symbol $C=C M U R(P(B E M / Q C R, \delta, c, \Psi), \overline{s p r}, \alpha)$. Taking into account conditions (25) and (26), we infer that the value $\pi\left(s_{j}\right)$ determined by Formula (23) is the probability of accurate transaction opening due to an observation of a current premise, $s_{j}$. Each accurately opened transaction is profitable for a speculator. Therefore, the probability $\pi\left(s_{j}\right)$ will be called the conditional success probability. In agreement with conditions (23) or (24), the ratio $\pi_{u p}$ is the infimum of conditional succes probabilities determined for acceptable transaction premise.

For any CMUR system, $C$, we define the success probability as the probability of accurate transaction opening due to use of this system. We get:

$$
\pi(C)=\frac{\sum_{s_{j} \in \mathbb{D}} p\left(s_{j}\right) \cdot \pi\left(s_{j}\right)}{\sum_{s_{j} \in \mathbb{D}} p\left(s_{j}\right)} .
$$

An expected single payment from transaction is defined as the expected value of payment obtained due to realization of this transaction on one lot of BEM. Then, by the symbol $\mathcal{Y}\left(s_{j}\right)$, we denote the expected single payment from transaction opened immediately after that the acceptable premise, $s_{j} \in \mathbb{D}$, has occurred. The expected single payment, $\mathcal{Y}\left(s_{j}\right)$, is equal to value

$$
\mathcal{Y}\left(s_{j}\right)=\left(2 \cdot \pi\left(s_{j}\right)-1\right) \cdot \delta-\overline{s p r}>0,
$$

expressed in pips [4].

Example 4 [4]. The transaction-making strategy implemented in $\operatorname{CMUR}(\mathcal{P}(X A G / U S D, 28,4, \Psi), 1,0.05)$ is presented in Table 3. All acceptable premises, $s_{j} \in \mathbb{D}(0.05,28,4,0.5179)=\mathbb{D}$, fulfilling condition (25) are marked by a star, *. Moreover, in Table 3, expected single payments, $\boldsymbol{y}\left(s_{j}\right)$, assesses benefits achieved by a speculator trading one lot of silver. 


\section{Effectiveness of the CMUR System}

The effectiveness of any trading system means being able to achieve the highest possible profits under conditions of the lowest possible risk. An important element of each trading process in any market is the proper choice of an effective supporting system. For realization of this choice, we can use criteria pointed out by Garcia et al. [44] and Li et al. [45], who point to the trading system features that should be evaluated. Their proposals are implemented for evaluating the CMUR system [4]. In further considerations, without loss of generality, we can ignore the leverage problem. All efficiency characteristics are determined for payment obtained due to realization of a single transaction on one lot of BEM. We assess the CMUR system effectiveness using the following notions.

Let a fixed CMUR system, $C=C M U R(\mathcal{P}(B E M / Q C R, \delta, c, \Psi), \overline{s p r}, \alpha)$, be given, with the space of all acceptable premises, $\mathbb{D}=\mathbb{D}\left(\alpha, c, \pi_{\text {up }}\right)$. The expected annual number of transactions is

$$
\mathcal{N}(C)=\sum_{s_{j} \in \mathbb{D}} \tilde{n}_{j}
$$

where $\tilde{n}_{j}$ is an annaualized number of the state, $s_{j}$, observations collected during construction of the prediction table $\mathcal{P}(B E M / Q C R, \delta, c, \Psi)$.

- $\quad$ The success probability, $\pi(C)$, is determined by the Formula (28)

- $\quad$ The expected unit payment, expressed in QCR units, is

$$
y(C)=10 \cdot[(2 \cdot \pi(C)-1) \cdot \delta-\overline{s p r}]
$$

- $\quad$ The expected unit profit is

$$
y(C)=\mathcal{N}(C) \cdot y(C)
$$

- The expected interest rate is

$$
i(C)=\frac{y(C)}{v_{B E M / Q C R}} \cdot 100 \%,
$$

where $v_{B E M / Q C R}$ is current Ask price of one lot of BEM.

In CMUR system evaluation, we understate the speculator's risk as the possibility of incurring losses as a result of placing an inaccurate order. In Reference [4], the risk index, $\mathcal{E}(C)$, is defined as expected Shannon's entropy [46] in the following way:

$$
\mathcal{E}(\mathcal{C})=\frac{-\sum_{s_{j} \in \mathbb{D}} p\left(s_{j}\right) \cdot\left(\pi\left(s_{j}\right) \cdot \ln \pi\left(s_{j}\right)+\left(1-\pi\left(s_{j}\right)\right) \cdot \ln \left(1-\pi\left(s_{j}\right)\right)\right)}{\ln 2 \cdot \sum_{s_{j} \in \mathbb{D}} p\left(s_{j}\right)}
$$

In summary, for the CMUR system, $C=C M U R(\mathcal{P}(B E M / Q C R, \delta, c, \Psi), \overline{s p r}, \alpha)$, its effectiveness should be characterized by a pair of

$$
(i(C), \mathcal{E}(C)) \text {. }
$$

The evaluation of effectiveness of the CMUR system can also be simplified by means of interest risk premium:

$$
\mathcal{B}(C)=\frac{i(C)}{\mathcal{E}(C)}
$$

The characteristics presented above are useful only for comparison of evaluated CMUR systems with other trading systems. 
Example 5 [4]. We evaluate effectiveness of the trading system CMUR(P $(X A G / U S D, 28,4, \Psi), 1,0.05)$ described in Examples 2, 3, and 4. On 28 December 2018, the silver price was $\$ 15.44$ per troy ounce [30]. It implies that for silver, the current lot value is as follows:

$$
v_{X A G / U S D}=1000 \mathrm{oz} \cdot \$ 15.44 / \mathrm{oz}=\$ 15,440 \text {. }
$$

The effectiveness characteristic of considered CMUR system are given in Table 4.

Table 4. Juxtaposition of effectiveness characteristics of $C M U R(\mathcal{P}(X A G / U S D, 28,4, \Psi), 1,0.05)$.

\begin{tabular}{cc}
\hline Characteristics & CMUR $(\mathcal{P}(X A G /$ USD, 28, 4, $\mathbf{\Psi}), \mathbf{1 , 0 . 0 5})$ \\
\hline Expected annual number of transactions & 187.4 \\
Success probability & 0.5763 \\
Expected unit payment & $\$ 34.34$ \\
Expected unit profit & $\$ 6435.31$ \\
Expected interest rate & $41.68 \%$ \\
Risk index & 0.9813 \\
Interest risk premium & 42.47 \\
\hline
\end{tabular}

Source: own calculations.

Effectiveness of the evaluated CMUR system is represented by the pair (41.68\%, 0.9813). The high interest rate of 41.68 tells us that the use of the system $\operatorname{CMUR}(\mathcal{P}(X A G / U S D, 28,4, \Psi), 1,0.05)$ on the gold market guarantees profits well above average. We can use the value 0.9813 of the risk index only for comparison with the system $C M U R(\mathcal{P}(X A G / U S D, 28,4, \Psi), 1,0.05)$ applied on the gold market with other trading systems applied on any exchange market.

\section{Optimization of the CMUR System}

The financial effectiveness increases along with the increase of expected interest and with the decrease of the systemic risk index. Therefore, the subset of all effective CMUR systems may be distinguished as the Pareto's optimum, determined as a two-criteria comparison of maximization of expected unit profit and minimization of risk index.

Let us take into account the single CMUR system. $C_{\delta, c}=C M U R(\mathcal{P}(B E M / Q C R, \delta, c, \Psi), \overline{s p r}, \alpha)$. To simplify further considerations, we can assume that the required unit return modulus, $\delta$, is an integer number. Therefore, we can restrict limit the search area for effective CMUR systems by the condition:

$$
\delta \in\left[\delta_{*}, \delta^{*}\right] \cap \mathbb{N}
$$

where

- $\delta_{*} \in \mathbb{N}$ is the least required unit return modulus, and

- $\delta^{*} \in \mathbb{N}$ is the greatest required unit return modulus.

Moreover, searching for the most effective CMUR system is always limited by condition

$$
c \in\left[c_{*}, c^{*}\right] \cap \mathbb{N}
$$

where

- $\quad c_{*} \in \mathbb{N}$ is the least assumed observation range, and

- $\quad c^{*} \in \mathbb{N}$ is the greatest assumed observation range.

The financial effectiveness of the CMUR system, $\mathcal{C}_{\delta, c}$, is characterized by the pair

$$
\left(i_{\delta, c}, \mathcal{E}_{\delta, c}\right)=\left(i\left(\mathcal{C}_{\delta, c}\right), \mathcal{E}\left(\mathcal{C}_{\delta, c}\right)\right)
$$


On the space of all compared CMUR systems, we determine some preorders, $C_{\delta, c} \cdot Q_{x} \cdot C_{\partial, f}$, which read:

$$
\text { "CMUR system } C_{\delta, c} \text { is not worse than CMUR system } C_{\partial, f} \text { " }
$$

In this section, we will apply the following preorders:

$$
\begin{gathered}
\mathcal{C}_{\delta, c} \cdot Q_{1} \cdot C_{\partial, f} \Leftrightarrow i_{\delta, c} \geq i_{\partial, f}, \\
\mathcal{C}_{\delta, c} \cdot Q_{2} \cdot C_{\partial, f} \Leftrightarrow \mathcal{E}_{\delta, c} \leq \mathcal{E}_{\partial, f},
\end{gathered}
$$

The set of all effective CMUR systems we appoint as Pareto's optimum are determined by multi-criterial comparison $Q_{1} \cap Q_{2}$. We solve this optimization task in two stages.

In the first stage, for each observation range, $c \in\left[c_{*}, c^{*}\right] \cap \mathbb{N}$, we execute the following algorithm: STEP 1:

$$
\mathbb{P}_{c}:=\left\{C_{\delta_{*}, c}\right\}
$$

STEP 2:

$$
\delta i:=\delta_{*}+1
$$

STEP 3:

$$
\begin{gathered}
\left(\forall_{C_{x, c} \in \mathbb{P}_{c}}:\left(i_{x, c} \geq i_{\delta, c} \wedge \mathcal{E}_{x, c} \geq \mathcal{E}_{\delta, c}\right) \vee\left(i_{x, c} \leq i_{\delta, c} \wedge \mathcal{E}_{x, c} \leq \mathcal{E}_{\delta, c}\right)\right. \\
\left.\vee\left(i_{x, c} \leq i_{\delta, c} \wedge \mathcal{E}_{x, c} \geq \mathcal{E}_{\delta, c}\right)\right) \\
\Downarrow \\
\mathbb{P}_{c}:=\mathbb{P}_{c} \cup\left\{\mathcal{C}_{\delta, c}\right\} .
\end{gathered}
$$

STEP 4:

$$
\forall_{C_{x, c} \in \mathbb{P}_{c}}:\left(\left(i_{x, c}<i_{\delta, c} \wedge \mathcal{E}_{x, c}>\mathcal{E}_{\delta, c}\right) \Rightarrow \mathbb{P}_{c}:=\mathbb{P}_{c} /\left\{C_{x, c}\right\}\right)
$$

STEP 5:

$$
\delta:=\delta+1
$$

STEP 6:

$$
\delta>\delta^{*} \Rightarrow \text { go to STOP. }
$$

STEP 7:

$$
\text { go to STEP } 3 .
$$

STOP.

In this way, we obtain the sequence $\left(\mathbb{P}_{c}\right)_{c=c_{*}}^{c^{*}}$ of partial optima of Pareto. In the second stage, we execute the following algorithm:

STEP 1:

$$
\mathbb{P}:=\mathbb{P}_{\mathcal{C}_{*}}
$$

STEP 2:

$$
c:=c_{*}+1
$$

STEP 3:

$$
\forall_{C_{x, c} \in \mathbb{P}_{c}}:\left(\begin{array}{c}
\left({ }_{C_{y, z} \in \mathbb{P}}:\left(i_{y, z} \geq i_{x, c} \wedge \mathcal{E}_{y, z} \geq \mathcal{E}_{x, c}\right) \vee\left(i_{y, z} \leq i_{x, c} \wedge \mathcal{E}_{y, z} \leq \mathcal{E}_{x, c}\right) \vee\left(i_{y, z} \leq i_{x, c} \wedge \mathcal{E}_{y, z} \geq \mathcal{E}_{x, c}\right)\right) \\
\Downarrow \\
P:=P \cup\left\{C_{x, c}\right\}
\end{array}\right)
$$

STEP 4:

$$
\forall_{C_{x, c} \in \mathbb{P}_{c}} \forall_{C_{y, z} \in \mathbb{P}}:\left(\left(i_{y, z}<i_{x, c} \wedge \mathcal{E}_{y, z}>\mathcal{E}_{x, c}\right) \Rightarrow \mathbb{P}:=\mathbb{P} /\left\{C_{y, z}\right\}\right)
$$


STEP 5:

$$
c:=c+1
$$

STEP 6:

$$
c>c^{*} \Rightarrow \text { go to STOP. }
$$

STEP 7:

$$
\text { go to STEP } 3 .
$$

STOP.

In this way, we obtain the Pareto's optimum, $\mathbb{P}$. Any CMUR system $C_{\delta, c} \in \mathbb{P}$ is an effective one. Then, the pair of control parameters $(\delta, c)$ is optimal.

\section{Case Study}

In this section, we explore the possibility of effective use of the CMUR system for speculations in exchange pair gold quoted in USD (XAU/USD). We have collected tick data containing 188,055,881 XAU/USD quotations from 31 December 2014 to 1 January 2020 [47]. For exchange pair XAU/USD, these tick data determine the Ask price trend. This trend graph is presented in Figure 2.

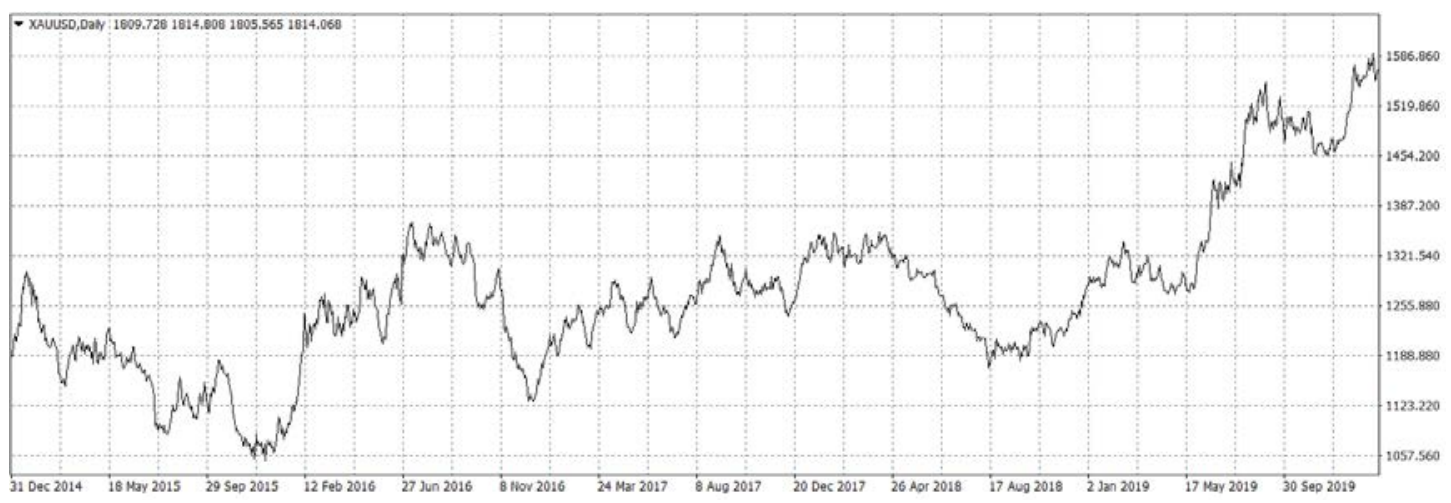

Figure 2. The graph for trend of Ask price of XAU/USD exchange pair.

We see that the trend $Q_{\text {Ask }}$ contains descriptions of bull market, bear market rand, and sideways market. For this reason, we can say that the collected tick data are representative.

On 2 January 2020, the average price of 1 gold ounce was $\$ 1520.30$. It means that average Ask price of 1 gold lot was $v_{X A U / U S D}=\$ 152,030$. Trading on the XAU/USD exchange market is managed by many brokers. We assume that the speculator gives orders to a broker who offers a spread of $\overline{s p r}=20$ pips. This level of spread is most often offered on the XAU/USD exchange market.

In our study, we evaluated all CMUR systems characterized by observation range $c=2,3,4,5$ and discretization unit $\delta=21,22, \ldots, 2420$. It means that we examined the effectiveness of 9680 variants of the CMUR system. We will lead our statistical research at the significance level $\alpha=0.05$. The assumed research area results from our experience gathered during previous studies [24-32].

For each pair of control parameters $(\delta, c)$, we determine following values and sets:

- $\mathcal{E}_{\delta, c}$ the binary representation containing all binary observations (5),

- $n_{\delta, c}$ the number of binary observations,

- $\Psi_{\delta, c}$ the training dataset (10),

- $a_{\delta, c}$ the average sample size given by the equation:

$$
a_{\delta, c}=\frac{n_{\delta, c}-c-1}{2^{c}}
$$

- $\mathcal{P}\left(X A G / U S D, \delta, c, \Psi_{\delta c)}\right.$ the prediction table (16), 
- $\mathbb{D}(0.05, \delta, c)$ the space of all well-justified premises (19),

- $\mathbb{D}_{\delta, c}=\mathbb{D}\left(0.05, \delta, c, \pi_{u p}\right)$ the space of all acceptable premises (24),

- $\mathcal{N}_{\delta, c}$ the expected annual number of transactions (29),

- $y_{\delta, c}$ the expected unit payment (31),

- $i_{\delta, c}$ the expected interest rate (33),

- $\mathcal{E}_{\delta, c}$ the risk index (34).

A sample size will be applied for heuristic evaluation of obtained solutions of the optimization task.

In the next step, we use the algorithm (43-49) for each observation range, $c=2,3,4,5$, separately. Obtained results are presented in Tables 5-8.

Table 5. Optimized variants of the CMUR system, $\operatorname{CMUR}(\mathcal{P}(X A U / U S D, \delta, 2, \Psi), 20,0.05)$

\begin{tabular}{ccccc}
\hline Discretization Unit $\delta$ & $\begin{array}{c}\text { Number of Binary } \\
\text { Representations } \boldsymbol{n}_{\boldsymbol{\delta}, \mathbf{2}}\end{array}$ & $\begin{array}{c}\text { Average Sample } \\
\text { Size } \boldsymbol{a}_{\boldsymbol{\delta}, \mathbf{2}}\end{array}$ & $\begin{array}{c}\text { Expected Interest } \\
\text { Rate } \boldsymbol{i}_{\boldsymbol{\delta}, \mathbf{2}}\end{array}$ & Risk Index $\mathcal{E}_{\delta, \boldsymbol{c}}$ \\
\hline 346 & 10,162 & 2540 & $36.10 \%$ & 0.994099 \\
528 & 4519 & 1129 & $38.58 \%$ & 0.994164 \\
\hline \multicolumn{5}{c}{ Source: Own elaboration. }
\end{tabular}

Table 6. Optimized variants of the CMUR system, $\operatorname{CMUR}(\mathcal{P}(X A U / U S D, \delta, 3, \Psi), 20,0.05)$.

\begin{tabular}{ccccc}
\hline Discretization Unit $\delta$ & $\begin{array}{c}\text { Number of Binary } \\
\text { Representations } \boldsymbol{n}_{\boldsymbol{\delta}, \boldsymbol{3}}\end{array}$ & $\begin{array}{c}\text { Average Sample } \\
\text { Size } \boldsymbol{a}_{\boldsymbol{\delta}, \mathbf{3}}\end{array}$ & $\begin{array}{c}\text { Expected Interest } \\
\text { Rate } \boldsymbol{i}_{\boldsymbol{\delta}, \mathbf{3}}\end{array}$ & Risk Index $\mathcal{E}_{\delta, \mathbf{3}}$ \\
\hline 375 & 8580 & 1072 & $55.90 \%$ & 0.991297 \\
376 & 8489 & 1061 & $59.37 \%$ & 0.993476 \\
463 & 5713 & 714 & $38.19 \%$ & 0.985322 \\
\hline
\end{tabular}

Source: Own elaboration.

Table 7. Optimized variants of the CMUR system, $\operatorname{CMUR}(\mathcal{P}(X A U / U S D, \delta, 4, \Psi), 20,0.05)$.

\begin{tabular}{ccccc}
\hline Discretization Unit $\boldsymbol{\delta}$ & $\begin{array}{c}\text { Number of Binary } \\
\text { Representations } \boldsymbol{n}_{\boldsymbol{\delta}, \boldsymbol{4}}\end{array}$ & $\begin{array}{c}\text { Average Sample } \\
\text { Size } \boldsymbol{a}_{\boldsymbol{\delta}, \mathbf{4}}\end{array}$ & $\begin{array}{c}\text { Expected Interest } \\
\text { Rate } \boldsymbol{i}_{\boldsymbol{\delta}, \mathbf{4}}\end{array}$ & Risk Index $\mathcal{E}_{\boldsymbol{\delta}, \boldsymbol{4}}$ \\
\hline 368 & 9021 & 564 & $55.02 \%$ & 0.987849 \\
375 & 8580 & 534 & $63.43 \%$ & 0.990001 \\
377 & 8498 & 531 & $66.08 \%$ & 0.991844 \\
404 & 7469 & 467 & $55.24 \%$ & 0.988295 \\
406 & 7385 & 461 & $38.28 \%$ & 0.987462 \\
418 & 6952 & 434 & $27.81 \%$ & 0.979963 \\
\hline
\end{tabular}

Source: Own elaboration.

Table 8. Optimized variants of the CMUR system, $\operatorname{CMUR}(\mathcal{P}(X A U / U S D, \delta, 5, \Psi), 20,0.05)$.

\begin{tabular}{ccccc}
\hline Discretization Unit $\boldsymbol{\delta}$ & $\begin{array}{c}\text { Number of Binary } \\
\text { Representations } \boldsymbol{n}_{\boldsymbol{\delta}, \mathbf{5}}\end{array}$ & $\begin{array}{c}\text { Average Sample } \\
\text { Size } \boldsymbol{i}_{\boldsymbol{\delta}, \mathbf{5}}\end{array}$ & $\begin{array}{c}\text { Expected Interest } \\
\text { Rate } \boldsymbol{i}_{\boldsymbol{\delta}, \mathbf{5}}\end{array}$ & Risk Index $\mathcal{E}_{\boldsymbol{\delta}, \mathbf{5}}$ \\
\hline 366 & 9047 & 283 & $89.91 \%$ & 0.985164 \\
406 & 7385 & 231 & $83.19 \%$ & 0.978913 \\
442 & 6268 & 196 & $88.70 \%$ & 0.98096 \\
513 & 4740 & 148 & $37.54 \%$ & 0.952324 \\
515 & 4734 & 148 & $29.28 \%$ & 0.920517 \\
517 & 4702 & 147 & $56.99 \%$ & 0.95236 \\
537 & 4252 & 133 & $66.33 \%$ & 0.96346 \\
& 4218 & 132 & $79.09 \%$ & 0.967076 \\
\hline
\end{tabular}

Source: Own elaboration.

It is very easy to see that if we use the optimal CMUR system linked to observation range $c=5$, then expected interest rates, $i_{\delta, 5}$, are not realistic. On the other hand, each average sample size, $i_{\delta, 5}$, 
is always low. Therefore, we can suppose that prediction tables $\mathcal{P}(X A U / U S D, \delta, 5, \Psi)$ are not reliable. For this reason, we propose to accept the value $a=300$ as the minimal average sample size. Of course, our proposal is only heuristic. The above heuristic inference leads to the omission of the optimal CMUR systems, $C M U R(\mathcal{P}(X A U / U S D, \delta, 5, \Psi), 20,0.05)$ in subsequent calculations.

In the last step, we use the algorithm (50-56) for all observation ranges, $c=2,3,4$, together. Obtained results are presented in Table 9.

Table 9. Effective variants of the CMUR system, $\operatorname{CMUR}(\mathcal{P}(X A U / U S D, \delta, c, \Psi), 20,0.05)$.

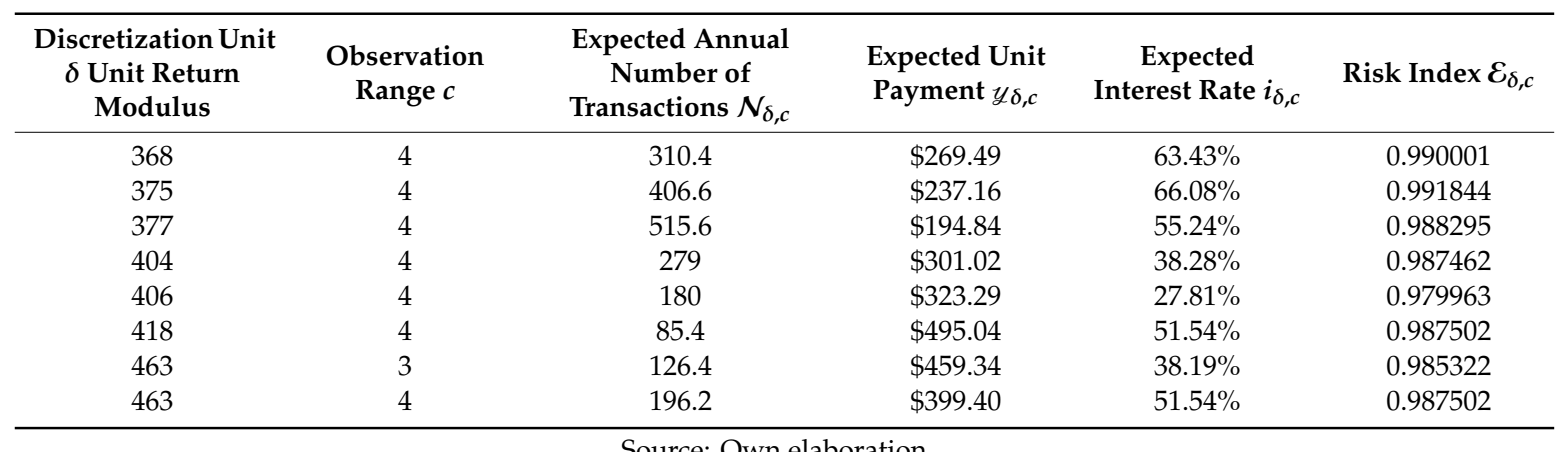

The above table can be used as follows: A speculator chooses one of the recommended values of unit return modulus. This choice implies a recommendation of the proper observation range. An interesting situation exists in the case of a recommendation unit return modulus $\delta=463$. Then, we have two options of choice:

- The observation range $c=3$ for speculators with risk aversion.

- The observation range $c=4$ for speculators with risk propensity.

This is a simple example of the CMUR system flexibility.

\section{Conclusions}

In this paper, we proposed an original method of selecting financially effective CMUR systems. Proper selection is given as Pareto' optimum determined by interest rate maximization and risk index minimization. In our case study, we show a heuristic limitation of optimization task. The results of the discussion point to further research directions.

Results obtained in the case study show that distinguished Pareto's optima are multi-element sets. This fact implies that a most-powerful parameter specification does not exist. This is a common situation in economics and finance. For this reason, in economics and finance, we are finding non-dominated parameter specification. It is a widely accepted approach in operations research. Therefore, such approach is applied in our paper.

Moreover, obtained results show that distinguished Pareto's optima are not convex. It implies that expected interest rate and risk index are not convex functions of CMUR system parameters. It implies that convex analysis cannot be used.

In our paper, we have used risk index (34) related to Shannon' entropy [46]. It would be useful to discuss the use of other suitable risk indices here. We propose to discuss the following ex-ante risk indices: inverse of success probability (28), single payment variance, and single payment semi-variance. In addition, we suggest taking max drawdown into account as ex-post risk indices. A rank correlation between these indices should be investigated. After this research, we will be able to start comparing CMUR systems and other types of trading systems. In the case study, we proposed to accept some values as minimal average sample size. This proposal is only heuristic. This means that there is a real need to build a formal method of determining minimal sample size. Additionally, we propose the following directions of investigation on the CMUR systems: 
- Influence of levering on CMUR system effectiveness, and

- Influence of lot value variability on systemic risk.

Author Contributions: Conceptualization, K.P. and M.D.S.; methodology, K.P.; data curation M.D.S.; validation, K.P. and M.D.S.; formal analysis, K.P.; resources, M.D.S.; writing—original draft preparation, K.P.; writing-review and editing, K.P. All authors have read and agreed to the published version of the manuscript.

Funding: This research received no external funding.

Acknowledgments: The authors are very grateful to the anonymous reviewers for their helpful suggestions and comments.

Conflicts of Interest: The authors declare no conflict of interest.

\section{References}

1. Kaldor, N. Essays on Economic Stability and Growth; The Free Press of Glencoe Published by Gerald Duckworth: Glencoe, IL, USA, 1960.

2. Huang, B.; Huan, Y.; Xu, L.D.; Zheng, L.; Zou, Z. Automated trading systems statistical and machine learning methods and hardware implementation: A survey. Enterp. Inf. Syst. 2019, 13, 132-144. [CrossRef]

3. Dymova, L.; Sevastjanov, P.; Kaczmarek, K. A Forex trading expert system based on a new approach to the rule-base evidential reasoning. Expert Syst. Appl. 2016, 51, 1-13. [CrossRef]

4. Piasecki, K.; Stasiak, M.D. The Forex Trading System for Speculation with Constant Magnitude of Unit Return. Mathematics 2019, 7, 623. [CrossRef]

5. Komunikat w Sprawie Wyników Osiaganych Przez Inwestorów na Rynku Forex. Available online: https: //www.knf.gov.pl/poprzednie_lata/komunikaty?articleId=53122\&p_id=18 (accessed on 2 January 2019).

6. News Releases AMF. 2014. Available online: https://www.amf-france.org/en_US/Actualites/Communiquesde-presse/AMF/annee_2014.html?docId=workspace\%3A\%2F\%2FSpacesStore\%2F96c52a14-3900-464f-8fff7d4700ff37e3 (accessed on 2 January 2019).

7. Aldridge, I. High-Frequency Trading: A Practical Guide to Algorithmic Strategies and Trading Systems, 2nd ed.; John Wiley and Sons: New York, NY, USA, 2013.

8. Petropoulos, A.; Chatzis, S.P.; Siakoulis, V.; Vlachogiannakis, N. A stacked generalization system for automated FOREX portfolio trading. Expert Syst. Appl. 2017, 90, 290-302. [CrossRef]

9. Bekiros, S.D. Heuristic learning in intraday trading under uncertainty. J. Empir. Financ. 2015, 30, 34-49. [CrossRef]

10. Dunis, C.; Zhou, B. Nonlinear Modelling of High Frequency Financial Time Series; John Wiley \& Sons: New York, NY, USA, 1998.

11. Dempster, M.A.; Leemans, V. An automated FX trading system using adaptive reinforcement learning. Expert Syst. Appl. 2006, 30, 543-552. [CrossRef]

12. Rundo, F.; Trenta, F.; Di Stallo, A.L.; Battiato, S. Advanced Markov-Based Machine Learning Framework for Making Adaptive Trading System. Computation 2019, 7, 4. [CrossRef]

13. Dempster, M.A.; Jones, C.M. A real-time adaptive trading system using genetic programming. Quant. Financ. 2001, 1, 397-413. [CrossRef]

14. Mendes, L.; Godinho, P.; Dias, J. A Forex trading system based on a genetic algorithm. J. Heuristics 2012, 18, 627-656. [CrossRef]

15. Evans, C.; Pappas, K.; Xhafa, F. Utilizing artificial neural networks and genetic algorithms to build an algo-trading model for intra-day foreign exchange speculation. Math. Comput. Model. 2013, 58, 1249-1266. [CrossRef]

16. Fischer, T.; Krauss, C. Deep learning with long short-term memory networks for financial market predictions. Eur. J. Oper. Res. 2018, 270, 654-669. [CrossRef]

17. Li, Y.; Zheng, W.; Zheng, Z. Deep robust reinforcement learning for practical algorithmic trading. IEEE Access 2019, 7, 108014-108022. [CrossRef]

18. Dixon, M.; Klabjan, D.; Bang, J.H. Classification-based financial markets prediction using deep neural networks. Algorithmic Financ. 2017, 6, 67-77. [CrossRef]

19. Sezer, O.B.; Ozbayoglu, A.M. Algorithmic financial trading with deep convolutional neural networks: Time series to image conversion approach. Appl. Soft Comput. 2018, 70, 525-538. [CrossRef] 
20. Rundo, F.; Trenta, F.; di Stallo, A.L.; Battiato, S. Grid trading system robot (GTSbot): A novel mathematical algorithm for trading FX market. Appl. Sci. 2019, 9, 1796. [CrossRef]

21. Alonso-Monsalve, S.; Suárez-Cetrulo, A.L.; Cervantes, A.; Quintana, D. Convolution on neural networks for high-frequency trend prediction of cryptocurrency exchange rates using technical indicators. Expert Syst. Appl. 2020, 149, 113250. [CrossRef]

22. Barbosa, R.P.; Belo, O. Multi-agent forex trading system. In Agent and Multi-Agent Technology for Internet and Enterprise Systems; Hakansson, A., Hartung, R., Nguyen, N.T., Eds.; Springer: Berlin/Heidelberg, Germany, 2010; pp. 91-118.

23. Aloud, M.; Fasli, M.; Tsang, E.; Dupuis, A.; Olsen, R. Modeling the High-Frequency FX Market: An Agent-Based Approach. Comput. Intell. 2017, 33, 771-825. [CrossRef]

24. Stasiak, M.D. Modelling of Currency Exchange Rates Using a Binary Representation. In Information Systems Architecture and Technology, Proceedings of the 37th International Conference on Information Systems Architecture and Technology_ISAT 2016, Karpacz, Poland, 18-20 September 2016; Wilimowska, Z., Borzemski, L., Grzech, A., Świątek, J., Eds.; Advances in Intelligent Systems and Computing; Springer: Berlin, Germany, 2016; Volume 524, pp. 153-161. [CrossRef]

25. Stasiak, M.D. Wave relations of exchange rates in binary-temporal representation. In Proceedings of the 35th International Conference Mathematical Methods in Economics MME 2017, Hradec Králové, Czech Republic, 13-15 September 2017; Prazak, P., Ed.; Gaudeamus, University of Hradec Kralove: Hradec Králové, Czech Republic, 2017; pp. 720-725.

26. Stasiak, M.D. Modelling of Currency Exchange Rates Using a Binary-Wave Representation. In Proceedings of 38th International Conference on Information Systems Architecture and Technology-ISAT 2017. Part III, Szklarska Poręba, Poland, 17-19 September 2017; Wilimowska, Z., Borzemski, L., Grzech, A., Świątek, J., Eds.; Springer: Berlin/Heidelberg, Germany, 2017; pp. 27-37. [CrossRef]

27. Stasiak, M.D. Modelling of Currency Exchange Rates Using a Binary-Temporal Representation. In Contemporary Trends in Accounting, Finance and Financial Institutions, Proceedings of the International Conference on Accounting, Finance and Financial Institutions (ICAFFI), Poznan, Poland, 19-21 October 2016; Choudhry, T., Mizerka, J., Eds.; Springer: Berlin/Heidelberg, Germany, 2018; pp. 97-110.

28. Stasiak, M.D. A study on the influence of the discretisation unit on the effectiveness of modelling currency exchange rates using the binary-temporal representation. Oper. Res. Dec. 2018, 28, 57-70.

29. Piasecki, K.; Stasiak, M. Exchange rate modelling with use of relative binary representation. In Proceedings of the 36th International Conference Mathematical Methods in Economics, Jindrichuv Hradec, Czech Republic, 12-14 September 2018; MatfyzPress, Publishing House of the Faculty of Mathematics and Physics Charles University: Prague, Czech Republic, 2018; pp. 410-415.

30. Piasecki, K.; Stasiak, M.; Staszak, Ż. Investment Decision Support on Precious Metal Market with Use of Binary Representation, Effective Investments on Capital Markets. In Proceedings of the 10th Capital Market Effective Investments Conference (CMEI 2018), Międzyzdroje, Poland, 19 September 2018; Tarczyński, W., Nermend, K., Eds.; Springer Proceedings in Business and Economics; Springer International Publishing AG: Cham, Switzerland, 2019; pp. 423-438. ISBN 978-3-030-21273-5. [CrossRef]

31. Stasiak, M. Trend analysis with use of binary representation. In Proceedings of the 37th International Conference Mathematical Methods in Economics MME 2019: Conference Proceedings, České Budějovice, Czech Republic, 11-13 September 2019; Houda, M., Remeš, R., Eds.; University of South Bohemia in České Budějovice, Faculty of Economics: České Budějovice, Czech Republic, 2019; pp. 529-535. ISBN 978-80-7394-760-6.

32. Piasecki, K.; Stasiak, M. Verification of the Precious Metals Market Effectiveness-Gold and Silver. In Proceedings of the International Scientific Conference Hradec Economic Days, Hradec Králové, Czech Republic, 2-3 April 2020; Jedlička, P., Marešová, P., Firlej, K., Soukal, I., Eds.; University of Hradec Králové: Hradec Králové, Czech, 2020; Volume 10, pp. 1-9. [CrossRef]

33. Chante, S. Beyond Technical Analysis: How to Develop and Implement a Winning Trading System; John Wiley \& Sons: New York, NY, USA, 1997.

34. Vastone, B.; Finnie, G. An empirical methodology for developing stock market trading systems using artificial neural networks. Expert Syst. Appl. 2009, 36, 6668-6680. [CrossRef]

35. Nelson, D.M.; Pereira, A.C.; De Oliveira, R.A. Stock market's price movement prediction with LSTM neural networks. In Proceedings of the International Joint Conference on Neural Networks, Anchorage, AK, USA, 14-19 May 2017; pp. 1419-1426. 
36. Chong, E.; Han, C.; Park, F.C. Deep learning networks for stock market analysis and prediction: Methodology, data representations, and case studies. Expert Syst. Appl. 2017, 83, 187-205. [CrossRef]

37. Borovkova, S.; Tsiamas, I. An Ensemble of LSTM Neural Networks or High-Frequency Stock Market Classification. Quant. Financ. 2019, 38, 600-619. [CrossRef]

38. Nakano, M.; Takahashi, A.; Takahashi, S. Bitcoin technical trading with artificial neural network. Phys. A Stat. Mech. Appl. 2018, 510, 587-609. [CrossRef]

39. Shintate, T.; Pichl, L. Trend Prediction Classification for High Frequency Bitcoin Time Series with Deep Learning. J. Risk Financ. Manag. 2019, 12, 17. [CrossRef]

40. De Villiers, V. The Point and Figure Method of Anticipating Stock Price Movements: Complete Theory and Practice; Stock Market Publications: New York, NY, USA, 1935.

41. Geisser, S. Predictive Inference; Chapman and Hall: New York, NY, USA, 1993.

42. Wallis, S.A. Binomial confidence intervals and contingency tests: Mathematical fundamentals and the evaluation of alternative methods. J. Quant. Linguist. 2013, 20, 178-208. [CrossRef]

43. Brown, L.D.; Cai, T.T.; Das Gupta, A. Interval Estimation for a Binomial Proportion. Stat. Sci. 2001, 16, 101-133. [CrossRef]

44. García, B.Q.; Gaytán, J.C.T.; Wolfskill, L.A. The Role of Technical Analysis in the Foreign Exchange Market. Glob. J. Bus. Res. 2012, 6, 17-22.

45. Li, W.; Wong, M.C.; Cenev, J. High frequency analysis of macro news releases on the foreign exchange market: A survey of literature. Big Data Res. 2015, 2, 33-48. [CrossRef]

46. Shannon, C.E. A Mathematical Theory of Communication. Bell Syst. Tech. J. 1948, 27, 379-423. [CrossRef]

47. Gold Reaches 6-Month Maximum. Available online: https://fbs.eu/analytics/news/gold-reaches-6-monthmaximum-4133 (accessed on 2 January 2019).

(C) 2020 by the authors. Licensee MDPI, Basel, Switzerland. This article is an open access article distributed under the terms and conditions of the Creative Commons Attribution (CC BY) license (http://creativecommons.org/licenses/by/4.0/). 